\title{
Dioxygen at Biomimetic Single Metal-Atom Sites: Stabilization or Activation? The Case of CoTPyP/Au(111)
}

\author{
F. Armillotta ${ }^{1,2} \odot$ A. Pividori ${ }^{1,3} \cdot$ M. Stredansky ${ }^{1,2} \cdot$ N. Seriani ${ }^{3} \cdot$ E. Vesselli ${ }^{1,2} \oplus$
}

Published online: 22 July 2020

(c) The Author(s) 2020

\begin{abstract}
By means of a combined experimental and computational approach, we show that a 2D metal-organic framework selfassembled at the $\mathrm{Au}(111)$ termination is able to mimic the $\mathrm{O}_{2}$ stabilization and activation mechanisms that are typical of the biochemical environment of proteins and enzymes. 5,10,15,20-tetra(4-pyridyl)21H,23H-porphyrin cobalt(III) chloride (CoTPyP) molecules on $\mathrm{Au}(111)$ bind dioxygen forming a covalent bond at the Co center, yielding charge injection into the ligand by exploiting the surface trans-effect. A weakening of the $\mathrm{O}-\mathrm{O}$ bond occurs, together with the development of a dipole moment, and a change in the molecule's magnetic moment. Also the bonding geometry is similar to the biological counterpart, with the $\mathrm{O}_{2}$ molecule sitting on-top of the Co atom and the molecular axis tilted by $118^{\circ}$. The ligand configuration lays between the oxo- and the superoxo-species, in agreement with the observed $\mathrm{O}-\mathrm{O}$ stretching frequency measured in situ at near-ambient pressure conditions.
\end{abstract}

Keywords ORR $\cdot$ CoTPyP $\cdot$ CoTPyP-fe $\cdot$ Oxygen activation $\cdot$ DFT $\cdot$ IR-vis SFG

\section{Introduction}

In a biomimetic perspective, single-atom catalysis is a promising route to design novel, cheap, efficient, selective, and stable heterogeneous catalysts [1]. In this context, tetrapyrrolic metallorganic frameworks (MOF) based on porphyrins or phthalocyanines tectons can represent an effective way to mimic the single metal atom active sites typical of many active enzymes or protein vectors that Nature exploits for many reactions, including oxygen conversion and transport [2-10]. In the specific case, the oxygen reduction reaction (ORR) is essential for life, as well as for many applicative

Electronic supplementary material The online version of this article (https://doi.org/10.1007/s11244-020-01333-9) contains supplementary material, which is available to authorized users.

E. Vesselli

evesselli@units.it

1 Physics Department, University of Trieste, via A. Valerio 2, 34127 Trieste, Italy

2 CNR-IOM, Laboratorio TASC, S.S. 14 km 163.5, Basovizza, 34149 Trieste, Italy

3 The Abdus Salam International Centre for Theoretical Physics, ICTP, Strada Costiera 11, 34151 Trieste, Italy purposes like e.g. the fuel cell related technologies. However, the relatively high stability of molecular oxygen requires the aid of catalytic materials or sites for the activation of the molecule [11-15]. The activity and the selectivity of natural systems are mainly determined by the metal species embedded in the active pocket site and, in a number of cases, the metal atom is coordinated in a tetrapyrrolic macrocycle. A fine tuning of the site's properties is granted by the local geometric and electronic configuration of the tetrapyrrole, including its residues: the proximal structure of the protein surroundings induces the well-known molecular trans-effect, while the distal structure operates through direct weak interactions $[2,16]$. As a matter of fact, the metal centers in biomimetic synthetic MOFs are coordinatively unsaturated, thus yielding available ligation sites for adducts and reactants [17-22]. Adsorption on surfaces provides not only a support for the molecules, but also stabilization due the interplay between the lateral and substrate interactions, as well as tuning of the charge transfer to or from the reaction site towards the substrate thanks to the surface trans-effect [23]. Thus, the surface, in principle very different from the biochemical environment of the natural system, manages to mimic some of the effects present there: the role played by the molecular trans-effect in the protein environment is taken up here by the surface trans-effect. Consequently, the 
fundamental comprehension of the electronic, magnetic, optical, and geometrical properties is essential for designing and engineering purposes, and surface science offers both highly controlled environments and atomic-scale resolution experimental techniques. However, most surface science experiments are performed under ultra-high vacuum conditions, i.e. under very different conditions from those of the biological system. This pressure gap, separating characterization and applicative environments, often strongly questions the significance of the results obtained by means of fundamental approaches. To this purpose, novel experimental techniques, such as Sum-Frequency Generation Spectroscopy, have been recently developed in order to bridge this gap, allowing investigations in situ and operando up to pressure values close to ambient conditions [2, 24-26].

Regarding the role played by the choice of the metal ion, Wurster et al. [17, 27] recently reported that the bimetallic MOF $\mathrm{M}_{1} \mathrm{TPyP}-\mathrm{M}_{2}\left(\mathrm{M}_{1}, \mathrm{M}_{2}=\mathrm{Fe}, \mathrm{Co}\right.$ and TPyP $=5,10,15,20$-tetrakis(4-pyridyl)porphyrin) can be selfassembled at the $\mathrm{Au}(111)$ termination showing a remarkable chemical stability and electrocatalytic activity with respect to both the oxygen reduction and evolution reactions. The peculiarity of this MOF is that, at variance with other similar cases $[18,28,29]$, in addition to the metal atom coordinated at the center of the macrocycle, a second metal species $\left(\mathrm{M}_{2}\right)$ can be embedded in the fourfold coordination sites formed by the pyridylic nitrogen atoms of neighboring molecules. Interestingly, the co-presence of two metal species yields nonlinear cooperative changes in catalytic activity, attributed to the peculiar electronic band dispersion that, on the basis of theoretical arguments, is expected to form within the 2D metallorganic crystal [30]. In particular, the presence of many frontier Dirac bands, corresponding to electronic states delocalized thorough the unit cell, could be responsible for the observed high electrocatalytic activity [30]. Moreover, the prediction of spin liquid states (in homometallic $\mathrm{M}_{1} \mathrm{TPyP}-\mathrm{M}_{1}$ ), long-range ferromagnetism, and half-metallicity (in heterometallic $\mathrm{M}_{1} \mathrm{TPy}-\mathrm{M}_{2}$ ) suggests interesting applicative perspectives in spintronics and high-temperature superconductivity [30-34].

Our present work focuses on the stabilization and the possible activation of the dioxygen molecule on the CoTPyP/ $\mathrm{Au}(111)$ network, which is a probable fundamental step in the ORR observed by Wurster et al. [17, 27]. We studied the system by means of in-situ near ambient pressure Infrared-Visible Sum-Frequency Generation spectroscopy (IR-Vis SFG), comparing the results with ab-initio calculations provided within the framework of Density Functional Theory (DFT). We have found that dioxygen is stabilized at the Co sites of the CoTPyP/Au(111) network at room temperature in $10^{-3} \mathrm{mbar}_{2}$. The $\mathrm{O}_{2}-\mathrm{Co}$ bond mainly involves the $\mathrm{O}_{2}\left(2 \pi^{*}\right)$ and the $\mathrm{Co}\left(3 d_{\mathrm{z}} 2\right)$ orbitals and yields bending of the adduct towards the $\mathrm{O}_{2}$ adsorption configuration typical of iron and cobalt tetrapyrrole-based sites of biological relevance [6]. Upon adsorption, the $\mathrm{O}-\mathrm{O}$ stretching frequency lowers due to the weakening of the intramolecular bond associated with the charge transfer, a molecular dipole is formed, and a $15 \%$ decrease in the porphyrin magnetization is observed, thus indicating stabilization and initial activation of the $\mathrm{O}_{2}$ molecule.

\section{Methods}

\subsection{Sample Preparation}

The Au(111) surface was cleaned by standard sputtering $\left(\mathrm{Ar}^{+}, 1.5 \mathrm{keV}\right)$ and annealing $(790 \mathrm{~K})$ cycles in UHV. The 5,10,15,20-tetra(4-pyridyl)21H,23H-porphyrin cobalt(III) chloride (CoTPyP) porphyrins were purchased from Frontier Scientific. The molecular source was a heated quartz crucible $(570 \mathrm{~K})$, the flux $(0.15 \mathrm{ML} / \mathrm{min})$ was monitored by means of a quartz microbalance, and long outgassing of the molecules in UHV up to about $530 \mathrm{~K}$ was necessary to get rid of the residual organic contaminants. The CoTPyP deposition at the $\mathrm{Au}(111)$ single crystal surface was performed with the sample kept at $500 \mathrm{~K}$ with a residual background pressure of $5 \times 10^{-10} \mathrm{mbar}$. The chlorine atom present at the Co site in the CoTPyP molecule detaches from the molecule and desorbs from the hot substrate $[17,35]$. The CoTPyPFe bimetallic network was obtained by self-assembly following the post-deposition of $1 \mathrm{ML}^{1}$ of the second metal (iron, $99.5 \%$ purity, resistively heated wire, $1.7 \mathrm{ML} / \mathrm{min}$ ) at the same substrate temperature, yielding spontaneous $\mathrm{Fe}$ coordination with the pyridyl groups in a fourfold geometry, as observed in previous STM measurements [17, 27]. The presence of the metal in the pyridylic site influences the lattice geometry of the MOF: CoTPyP porphyrins arrange, when adsorbed on $\mathrm{Au}(111)$, in a $2 \mathrm{D}$ oblique lattice with vector lengths of 1.45 and $1.50 \mathrm{~nm}$, respectively, forming an angle of $85^{\circ}$. The presence of the additional $\mathrm{M}_{2}$ iron atom, in the case of CoTPyP-Fe, re-arranges the superstructure, yielding a rectangular lattice with the same former vector lengths [17].

\subsection{IR-Vis SFG}

IR-Vis Sum Frequency Generation vibronic spectroscopy is a nonlinear optical technique $[26,36]$ The measurements were performed in a dedicated setup [37]. A UHV

\footnotetext{
$\overline{11 \mathrm{ML} \text { with }}$ respect to porphyrin layer, 0.03 ML with respect to $\mathrm{Au}(111)$ substrate.
} 
system with a base pressure of $5 \times 10^{-11}$ mbar hosting standard surface science preparation and characterization techniques is directly coupled with a high-pressure cell for in situ IR-Vis SFG spectroscopy. The reactor is equipped with a gas system to handle the reactants' pressure in the $10^{-9}-10^{+2}$ mbar range. The inlet and outlet of the laser beams are provided by UHV-compatible $\mathrm{BaF}_{2}$ windows. The $\mathrm{Au}(111)$ disc was mounted on Ta wires, also used for resistive heating. The excitation source (Ekspla, $1064 \mathrm{~nm}, 30 \mathrm{ps}, 50 \mathrm{~Hz})$ delivers a $532 \mathrm{~nm}(2.33 \mathrm{eV}) \mathrm{sec}-$ ond harmonic visible beam and IR radiation, tunable in the $1000-4500 \mathrm{~cm}^{-1}$ range. The spectra are firstly normalized to the IR and Vis pumps intensities, and then to a reference spectrum of clean Au to take into account the rotovibrational contributions of $\mathrm{H}_{2} \mathrm{O}$ and $\mathrm{CO}_{2}$ along the optical path, depending on the energy range. The gold substrate notoriously contributes with an intense nonresonant SFG signal [38-40], thus normalization of the data and counting statistics issues (signal to noise ratio) have to be rigorously accounted for. The normalized SFG spectra were analyzed by least-squares fitting to a parametric, effective expression of the nonlinear second-order susceptibility [26, 41, 42]. The expression (1) well reproduces the observed lineshapes, accounting for the resonant IR-Vis vibronic transitions and for the nonresonant background, and describing all the interference terms:

$\frac{I_{S F G}\left(\omega_{I R}\right)}{I_{V I S} I_{I R}\left(\omega_{I R}\right)} \propto\left|A_{N \operatorname{Res}}+\sum_{k} \frac{A_{k} e^{i \Delta \varphi_{k}}}{\omega_{I R}-\omega_{k}+i \Gamma_{k}}\right|^{2}$

$\mathrm{A}_{\mathrm{NRes}}$ and $\mathrm{A}_{\mathrm{k}}$ account for the amplitudes of the nonresonant and $\mathrm{k}^{\text {th }}$-resonant contributions, respectively. $\Delta \varphi_{\mathrm{k}}$ is the phase difference between the kth-resonant and nonresonant signals. $\omega_{\mathrm{k}}$ is the energy position of the line and $\Gamma_{\mathrm{k}}$ its Lorentzian broadening, related to the dephasing rate, which in turn stems from the energy lifetime and the elastic dephasing of the excited vibronic state [43]. In the manuscript, we plot the normalized IR-Vis SFG signal intensity (black dots), together with the best fit (black lines) and the deconvolution of each resonance with its interference with the nonresonant background (color-filled curves). The latter are calculated with the parameters obtained from the fitting procedure following:

$\frac{I_{S F G, k}\left(\omega_{I R}\right)}{I_{V I S} I_{I R}\left(\omega_{I R}\right)} \propto\left|A_{N R e s}+\frac{A_{k} e^{i \Delta \varphi_{k}}}{\omega_{I R}-\omega_{k}+i \Gamma_{k}}\right|^{2}$

These plots interestingly put in direct evidence the amplitude and the relative phase for each of the resonances. Further details and examples can be found in our previous work [21, 22, 41]. In the present study, all spectra were collected in the ppp polarization configuration (SFG-visible-infrared).

\subsection{DFT}

The electronic-structure calculations were performed in the framework of Density Functional Theory (DFT) in the spin-polarized version, as implemented in the Quantum ESPRESSO suite of codes [44, 45]. Vanderbilt ultrasoft pseudopotentials were employed [46], together with a plane-wave basis set with energy cutoffs of $50 \mathrm{Ry}$ for the wavefunctions and 500 Ry for the charge density. The large simulation cell with a size of $25 \AA$ made it possible to obtain $a b$ accurate description of the system using only one k-point (the Gamma point) to sample the Brillouin zone. The Perdew-Burke-Ernzerhof (PBE) functional was used to describe exchange and correlation [47], together with a Hubbard U correction applied to the $3 d$ states of cobalt [48]. A value of $\mathrm{U}=4.4 \mathrm{eV}$ was chosen following the literature [49]. A Methfessel-Paxton scheme was used for the occupation of the electronic states, with a smearing of $0.02 \mathrm{Ry}$. Van der Waals interactions were included through the Grimme DFTD3 approach [50]. The system was relaxed until the forces were smaller than $10^{-3}$ a.u. All calculated atomic charges mentioned in the paper are Löwdin charges [51].

\section{Results and Discussions}

In a first step, the CoTPyP/Au(111) monolayer was characterized by means of IR-Vis SFG spectroscopy. In the $1550-1620 \mathrm{~cm}^{-1}$ energy range (Fig. S1), vibronic resonances are observed at $1560,1570,1589$, and $1595 \mathrm{~cm}^{-1}$. They are associated with the $\nu\left(\mathrm{C}_{\beta}-\mathrm{C}_{\beta}\right)$ stretching modes internal to the macrocycle and with the $\delta$ (py) bending mode of the pyridyl groups (for details about the carbon atom notation see Fig. S2) [52-54]. In a first approximation, the dephasing time of these excitations has been assumed to be the same, thus reasonably reducing the number of free fitting parameters. At lower energies, from 1200 to $1300 \mathrm{~cm}^{-1}$ (Fig. 1a, bottom), we observe other resonances, attributed to the $\delta($ py) modes at $1215 \mathrm{~cm}^{-1}$, to a combination of $\nu\left(\mathrm{C}_{\alpha}-\mathrm{N}\right)+\delta\left(\mathrm{C}_{\beta}-\mathrm{H}\right)$, $\nu\left(\mathrm{C}_{\mathrm{m}}\right.$-pyridyl $)$, and $\delta(\mathrm{C}-\mathrm{H})$ modes at $1233 \mathrm{~cm}^{-1}$, and to $\delta\left(\mathrm{C}_{\mathrm{m}}-\right.$ py), $\nu\left(\mathrm{C}_{\alpha}-\mathrm{N}\right)$, and $\delta(\mathrm{py})$ modes at $1264 \mathrm{~cm}^{-1}$. The assignment of the observed resonances to the corresponding vibrational modes is reported in Table 1, while the quantitative details of the best fitting profiles are reported in the Supporting Information (Table S1). Interestingly, for the CoTPyP-Fe monolayer, no quantitative spectroscopic differences have been observed in the $1200-1300 \mathrm{~cm}^{-1}$ range.

A stepwise annealing experiment of the CoTPyP layer deposited at room temperature (Fig. S3) provided the necessary information to establish the temperature stability of the framework and the optimal deposition temperature for best ordering on the $\mathrm{Au}(111)$ surface. While the nonresonant background does not change with the stepwise annealing 

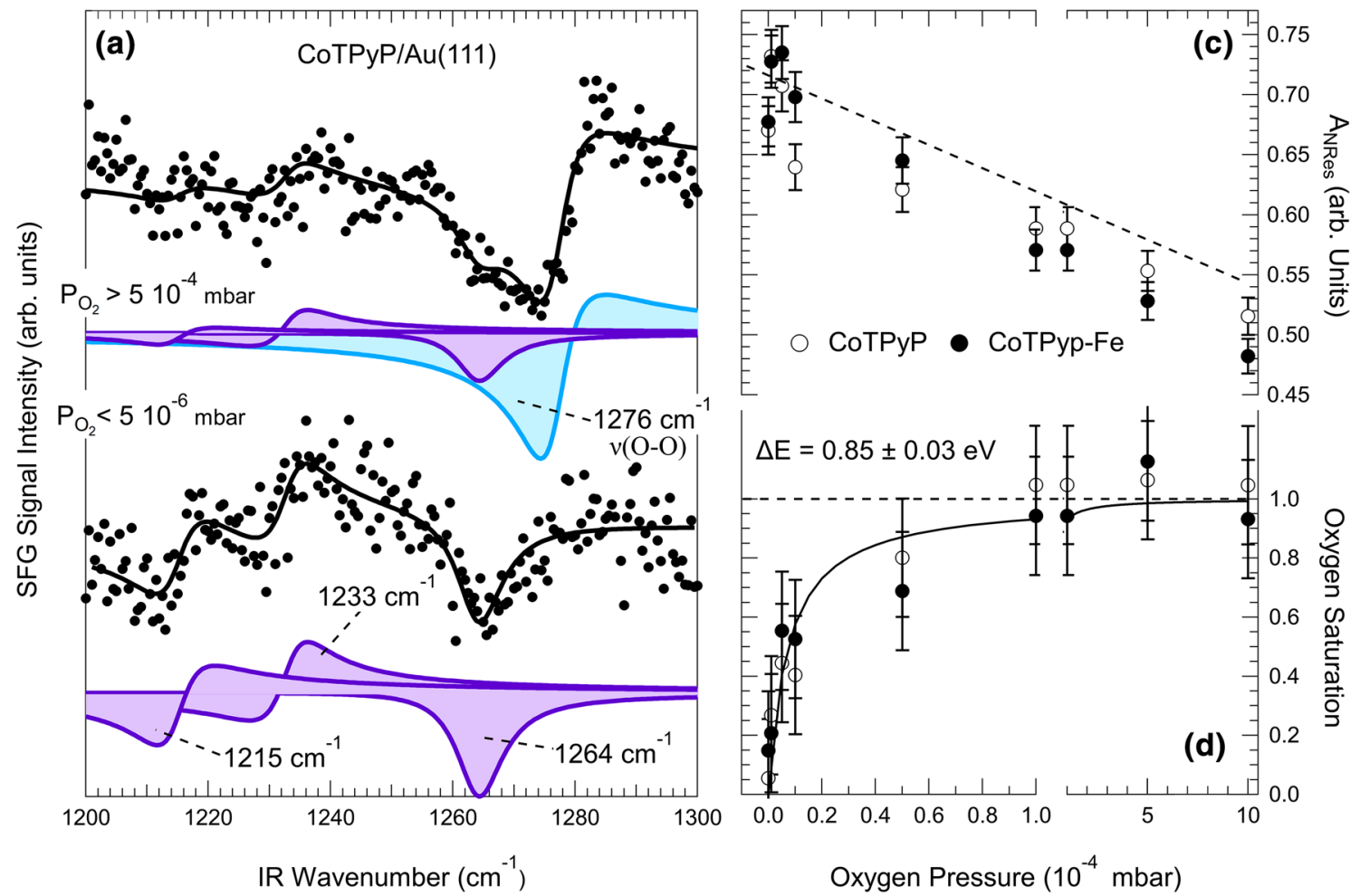

Fig. 1 a Normalized IR-vis SFG spectra (black dots) of 1.0 ML CoT$\mathrm{PyP} / \mathrm{Au}(111)$ in Ultra-High Vacuum (bottom) and in oxygen pressure (top), together with the best fit (solid black line) and the deconvolution of the resonances (filled profiles); b oxygen saturation curve (black and white dots) obtained from the SFG resonant amplitude of

Table 1 Positions of the vibronic resonances and comparison with the literature, yielding the assignment with the corresponding vibrational modes. Standard notation for carbon atoms is used (Fig. S2)

\begin{tabular}{llll}
\hline This work & Literature & References & Mode assignment \\
\hline 1215 & $1214-1219$ & {$[52-54]$} & $\delta($ py $)$ \\
1233 & $1224-1238$ & {$[76,77]$} & $\nu\left(\mathrm{C}_{\alpha}-\mathrm{N}\right)+\delta\left(\mathrm{C}_{\beta}-\mathrm{H}\right)$ \\
& $1227-1259$ & {$[77]$} & $\nu\left(\mathrm{C}_{\mathrm{m}}-\mathrm{Phenyl}\right)$ \\
& $1215-1237$ & {$[78]$} & $\delta(\mathrm{C}-\mathrm{H})$ \\
1264 & 1255 & {$[53]$} & $\delta\left(\mathrm{C}_{\mathrm{m}}-\mathrm{py}\right), \nu\left(\mathrm{C}_{\alpha}-\mathrm{N}\right), \delta(\mathrm{py})$ \\
& $1252-1253$ & {$[52,54]$} & $\delta\left(\mathrm{C}_{\mathrm{m}}-\mathrm{py}\right)$ \\
1276 & & & $\nu(\mathrm{O}-\mathrm{O})$ \\
1560 & $1554-1565$ & {$[52,54]$} & $\nu\left(\mathrm{C}_{\beta}-\mathrm{C}_{\beta}\right)$ \\
1570 & 1576 & {$[53]$} & $\nu\left(\mathrm{C}_{\beta}-\mathrm{C}_{\beta}\right)$ \\
1589 & 1576 & {$[53]$} & $\nu\left(\mathrm{C}_{\beta}-\mathrm{C}_{\beta}\right)$ \\
& $1609-1641$ & {$[52]$} & $\delta(\mathrm{py})$ \\
1595 & $1594-1641$ & {$[52,54]$} & $\delta(\mathrm{py})$ \\
\hline
\end{tabular}

temperature, a general decrease of the resonant amplitudes is observed above $500 \mathrm{~K}$ (Fig. S4), in agreement with the literature [17]. Thus, the best CoTPyP monolayer is obtained upon deposition of the molecules with the substrate kept at $500 \mathrm{~K}$. the mode at $1276 \mathrm{~cm}^{-1}$ by a fit with a Langmuir uptake model (solid line); white dots refer to CoTPyP/Au(111) system, while black ones to the bimetallic CoTPyP-Fe/Au(111) layer; c amplitude of the nonresonant background as a function of the $\mathrm{O}_{2}$ background pressure (the dashed line is drawn to guide the eye)

The monolayer obtained following this recipe was then exposed to increasing background pressures of molecular oxygen at room temperature. IR-Vis SFG spectra were collected in situ. A resonant feature progressively grows at $1276 \mathrm{~cm}^{-1}$ with $\mathrm{O}_{2}$ pressure (Fig. 1a, top, cyan deconvolution), reversibly disappearing when recovering UHV conditions (Fig. S5). This feature starts to grow at about $\mathrm{P}_{\mathrm{O} 2}=1 \times 10^{-5}$ mbar and reaches its maximum amplitude above $\mathrm{P}_{\mathrm{O} 2}=5 \times 10^{-4}$ mbar (Figs. $1 \mathrm{~b}$ and S6a). We also find that the tetrapyrroles are not significantly further distorted upon exposure to oxygen since the phases associated with the corresponding SFG resonances do not evolve. Interestingly, the resonance at $1276 \mathrm{~cm}^{-1}$ lays in between the $\nu(\mathrm{O}-\mathrm{O})$ stretching frequency of an adsorbed superoxide $\left(\mathrm{O}_{2}{ }^{-}\right)$species, as in the case of $\mathrm{O}_{2}$ chemisorbed on $\mathrm{Ag}(001)$ $\left(1049 \mathrm{~cm}^{-1}\right)[55,56]$, and that of the physisorbed/gaseous $\mathrm{O}_{2}$ (1549 and $1580 \mathrm{~cm}^{-1}$, respectively [56-58]). For larger charge transfer (more than $1 \mathrm{e}^{-}$), as for example for the peroxide species $\left(\mathrm{O}_{2}{ }^{2-}\right)$, an even lower stretching frequency would be expected $\left(637-677 \mathrm{~cm}^{-1}[59,60]\right)$. The $v(\mathrm{O}-\mathrm{O})$ mode that we observe at $1276 \mathrm{~cm}^{-1}$ is also in remarkable agreement with what observed on similar tetrapyrrole-based compounds, where $1278 \mathrm{~cm}^{-1}$ and $1247 \mathrm{~cm}^{-1}$ were found for 
$\mathrm{O}_{2}$-CoTPP and $\mathrm{O}_{2}$-CoPc, respectively $[29,58,61]$. Our finding suggests therefore that $\mathrm{O}_{2}$ binding and stabilization at the CoTPyP molecule on $\mathrm{Au}(111)$ occur via a charge transfer mechanism to the ligand of a fraction of an $\mathrm{e}^{-}$, yielding an adsorbed $\mathrm{O}_{2}{ }^{\delta-}$ species, bound to the Co atom. This picture is confirmed by our DFT calculations reported in the following. We mention here that the bimetallic CoTPyP-Fe layer shows a similar behavior (Fig. S6b), with the same vibrational resonance growing as a function of the $\mathrm{O}_{2}$ background pressure. This indicates that both the monometallic $\mathrm{Co}$ and bimetallic Co-Fe porphyrin monolayers behave in the same way with respect to oxygen adsorption, at least within the investigated pressure range. At the solid-liquid interface under electrochemistry conditions, for the CoT$\mathrm{PyP}-\mathrm{Fe} / \mathrm{Au}(111) \mathrm{MOF}$ the turnover frequency (TOF) for the ORR is larger by two orders of magnitude with respect to the case of CoTPyP/Au(111), suggesting a synergic role of the additional $\mathrm{Fe}$ atoms $[17,27]$. In our work, we show that the molecule interacts more strongly with the Co centers where the first step of charge injection takes place, thus suggesting that a cooperative mechanism involves the neighboring $\mathrm{Fe}$ sites for the successive steps of the reaction.

The evolution of the amplitude of the resonance at $1276 \mathrm{~cm}^{-1}$, as obtained from the deconvolution procedure, has been exploited for the evaluation of the interaction strength between oxygen and the metallorganic monolayer. The amplitude-pressure uptake curve has been fitted according to a rate model. The IR-Vis SFG spectra of the oxygen uptake (Fig. S6) have been fitted by releasing only the amplitudes of the resonant and nonresonant signals, as a first approximation to avoid overfitting with low statistics, while the lineshape parameters were obtained from a global fit of the whole data set. The results are reported in Fig. 1bc: while the resonant amplitude grows with the oxygen background pressure (Fig. 1b), the nonresonant contribution to the SFG signal progressively diminishes (Fig. 1c), witnessing substantial changes in the electronic configuration of the system close to the Fermi level $\left(\lambda_{\text {Vis }}=532 \mathrm{~nm}, v\right.$ $\mathrm{h} \nu=2.33 \mathrm{eV}$ ) during the $\mathrm{O}_{2}$ uptake. We model the saturation dependence on the $\mathrm{O}_{2}$ pressure at equilibrium by equaling adsorption and desorption rates according to the following rate equation:

$$
0=\frac{d \theta\left(P_{O_{2}}\right)}{d t}=\frac{P_{O_{2}} s_{0}\left(1-\theta\left(P_{O_{2}}\right)\right)}{\sqrt{2 \pi m_{O_{2}} k_{B} T_{G A S}}}-v_{0} \theta\left(P_{O_{2}}\right) e^{-\frac{\Delta E}{k_{B} T_{S U R F}}}
$$

$\left(\mathrm{s}_{0}\right.$ : sticking coefficient; $\nu_{0}$ : pre-exponential factor; $\left.\mathrm{T}_{\mathrm{GAS}}=\mathrm{T}_{\mathrm{SURF}}=300 \mathrm{~K}\right)$. The first term accounts for the dioxygen impinging flux (Knudsen factor) and sticking coefficient, and is proportional to the number of free cobalt sites $(1-\theta)$ for non-dissociative adsorption. The second term accounts for the desorption rate and is proportional to the Boltzmann factor including the desorption barrier $\Delta \mathrm{E}$. The two strong but necessary assumptions are $\mathrm{s}_{0}=1$ and $\nu_{0}=10^{+13} \mathrm{~s}^{-1}$ [62]. Changing the pre-exponential $\nu_{0}$ by two orders of magnitude affects the desorption energy by $\pm 15 \%$. Equation 3 can be easily solved for $\theta$. We assume that $\theta$ is proportional to the experimentally measured amplitude of the oxygen-related resonance at $1276 \mathrm{~cm}^{-1}$. This is reasonable since we expect that no relevant dipole-induced nonlinear effects take place due to the large distance between the single Co sites (of the order of $15 \AA[17,27])$. By least-square fitting of the experimental uptake curve, according to the model, we obtain $\Delta \mathrm{E}=0.85 \pm 0.03 \mathrm{eV}$. We notice that the model relies on a single free fitting parameter, i.e. the desorption energy. Nevertheless, it reproduces the data remarkably.

DFT calculations confirm and support the experimental findings. We performed simulations of $\mathrm{O}_{2}$ interacting with the CoTPyP molecule to investigate the adsorption geometry, the adsorption energy, the charge redistribution effects, and the formation of the dipole within the $\mathrm{O}_{2}$ molecule that is necessary to generate the SFG signal. The presence of the $\mathrm{Au}(111)$ substrate has been simulated with an $\mathrm{Au}_{13}$ nanocluster, on the basis of chemical and geometrical arguments, in order to minimize the required computational resources with respect to the case of the full Au surface termination. The $\mathrm{Au}_{13}$ nanocluster is often exploited in similar cases since it reproduces most of the effects associated with the presence of a gold substrate [63]. The cluster was relaxed and optimized separately, and its atomic coordinates were then fixed in order to resemble the bulk rigidity, so further reducing the computational time. The purpose of this model is to show the effect of a gold support on the adsorption properties of the molecules, although clearly the geometry will not be reproduced in all fine details. After relaxation in the gas-phase, the CoTPyP molecule has been added with the $\mathrm{Co}$ atom put on-top with respect to $\mathrm{Au}_{13}$ cluster (Fig. 2). The porphyrin then relaxes to a configuration where the Co-Au distance is $2.63 \AA$ [16, 64]. With respect to the gasphase configuration, the $\mathrm{C}-\mathrm{N}$ and $\mathrm{C}-\mathrm{C}$ distances in the macrocycle are augmented by 0.02 and $0.08 \AA$, respectively. The molecule almost maintains its fourfold symmetry, with unrotated pyridyl rings, while the dihedral angles between the molecular plane and the pyridylic/pyrrolic groups are $+20^{\circ} /-20^{\circ}$, respectively (as in the gas phase). In the literature [16, 64-66], ordered monolayers of similar systems show conformational adaptation to the flat underlying surface, resulting in a two-fold symmetry due to a saddleshape deformation of the macrocycle with the residues rotated by $16^{\circ}$ to $30^{\circ}$, and tilted by about $40^{\circ}$. Interestingly, it is well-known that these deformations strongly contribute in the reactivity of the whole macrocycle with respect to adsorption and/or activation of ligands [67]. We have therefore to consider that in our simulation the replacement of the $\mathrm{Au}(111)$ termination with the $\mathrm{Au}_{13}$ cluster results in a 

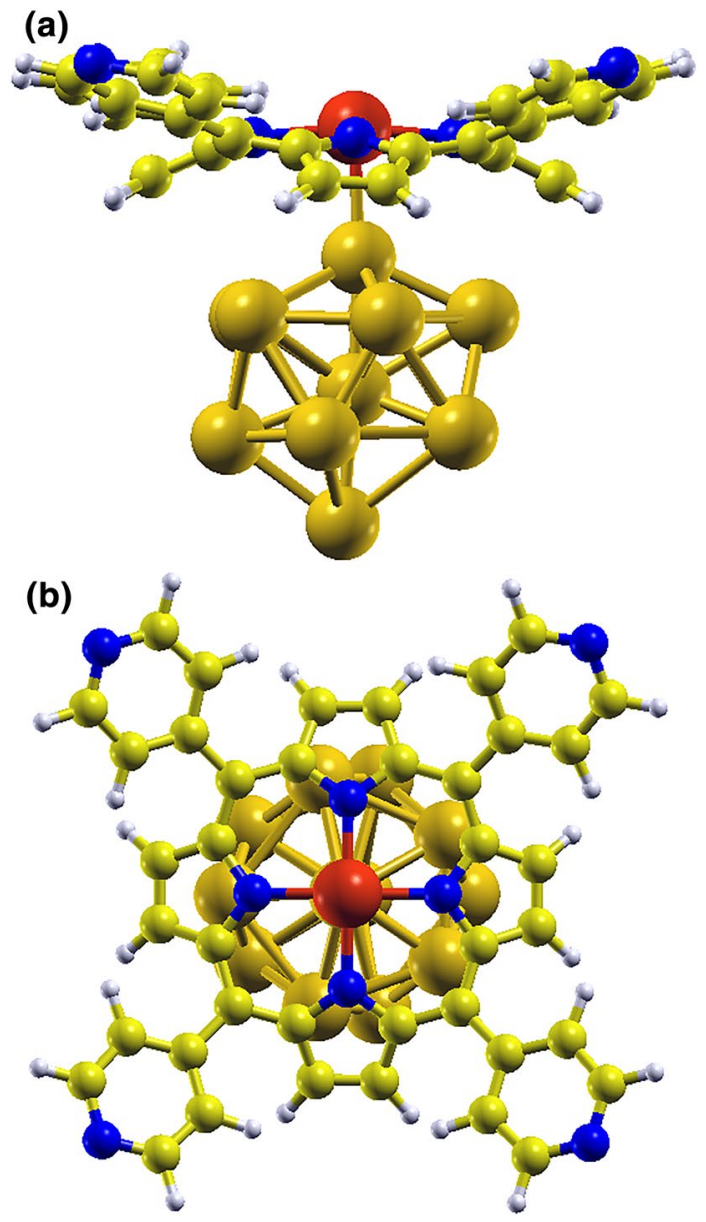

Fig. 2 Side (a) and top (b) views of the calculated CoTPyP adsorption geometry on the $\mathrm{Au}_{13}$ nanocluster; color legend: yellow (C), white $(\mathrm{H})$, blue $(\mathrm{N})$, gold $(\mathrm{Au})$, red $(\mathrm{Co})$

weaker gold interaction with the molecule's periphery. Nevertheless, since the goal of the simulation was to get insight into the $\mathrm{O}_{2}-\mathrm{Co}-\mathrm{Au}$ bond and the related charge redistribution trans-effects, we adopted the computationally cheaper $\mathrm{Au}$ cluster configuration that allowed the correct reproduction of the electronic configuration of the system, in agreement with previous literature on the full $\mathrm{Au}(111)$ termination [68]. The calculated spin-polarized projection densities of states (SP-PDOS) of Co $3 d_{\mathrm{z}} 2$ orbital are plotted in Fig. 3 for the CoTPyP in the gas-phase (d) and bonded to the $\mathrm{Au}_{13}$ cluster (b). In the latter case, upon adsorption on $\mathrm{Au}$, the $\mathrm{Co}$ atom in the macrocycle loses $0.39 \mathrm{e}^{-}$in favor of the Au cluster, thus the Co atom is in between a Co(II) and a Co(III) species, in agreement with the literature $[17,69]$. The Co $3 d$ orbitals are split and broadened due to the crystal field effect and the Co-substrate coupling. We focus now our discussion on the out-of-plane Co $3 d_{\mathrm{z}} 2,3 d_{\mathrm{xz}}$, and $3 d_{\mathrm{yz}}$ orbitals that are involved in the following step, i.e. $\mathrm{O}_{2}$ ligation. The $3 d_{\mathrm{z}} 2$ orbital is half-empty in the gas-phase (Fig. $3 \mathrm{c}$ and $3 \mathrm{~d}$ ) and shifts below the Fermi level upon adsorption on $\mathrm{Au}$

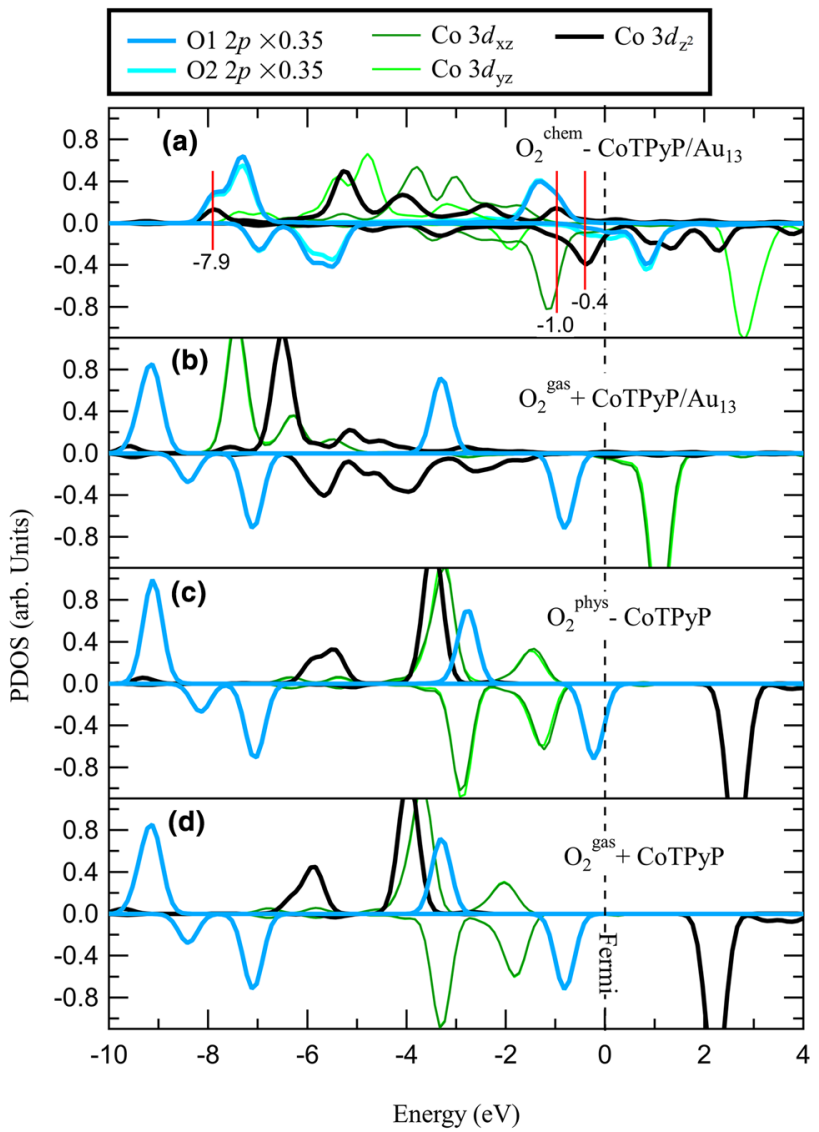

Fig. 3 Projected density of states for the $\mathrm{Co}$ and $\mathrm{O}$ atoms for a $\mathrm{O}_{2}$-CoTPyP/Au $\mathrm{Au}_{13}$, b gas phase $\mathrm{O}_{2}+\mathrm{CoTPyP} / \mathrm{Au}_{13}, \mathbf{c ~} \mathrm{O}_{2}-\mathrm{CoTPyP}$ with no $\mathrm{Au}_{13}$ cluster, and $\mathbf{d}$ gas phase $\mathrm{O}_{2}+\mathrm{CoTPyP}$ with no $\mathrm{Au}_{13}$ cluster. $\mathrm{O} 1$ refers to the proximal oxygen, while $\mathrm{O} 2$ to the distal one. Evidence for the formation of the $\mathrm{O}_{2}-\mathrm{Co}\left(3 d_{\mathrm{z}} 2\right)$ chemical bonding due to orbital overlap is indicated by the red lines in a spin-up bonding states at 7.9 and $1.0 \mathrm{eV}$, and spin-down bonding at $0.4 \mathrm{eV}$ below the Fermi level

(Fig. 3b), thus becoming entirely filled. The Co $3 d_{\mathrm{xz}}$ and $3 d_{\mathrm{yz}}$ are filled and degenerate in the gas-phase, but undergo deep modifications upon binding to $\mathrm{Au}$. The spin-down channel shifts by more than $2 \mathrm{eV}$ above Fermi, thus becoming empty. This is a crucial point in order to get insight into the $\mathrm{O}_{2}$ ligation mechanism $[6,70,71]$, which, as we will see, will involve the whole Co $3 d$ configuration and not just the $3 d_{\mathrm{z}} 2$ as recently and differently prospected in [68]. When the dioxygen molecule is let interact with the CoTPyP/Au ${ }_{13}$ cluster (Fig. 3a), it binds to the Co site with an energy of $0.68 \mathrm{eV}$, thus in agreement with the experimental value of $0.85 \pm 0.03 \mathrm{eV}$ when considering systematic errors originating from the stringent assumptions in the fitting model (sticking coefficient, pre-exponential factor). Indeed, when the same simulation is performed without the Au cluster, the trans-effect does not take place and we get only weak $\mathrm{O}_{2}$-Co physisorption, yielding binding energy values of the order 

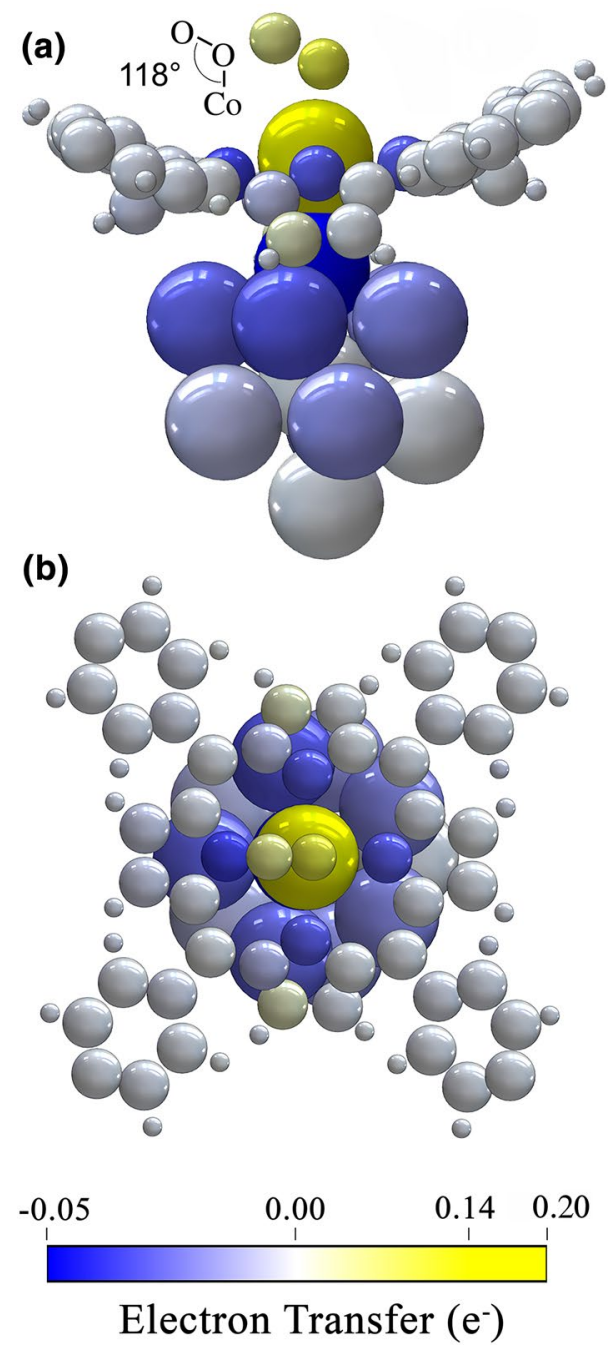

Fig. 4 Side (a) and top (b) view of the optimized $\mathrm{O}_{2}-\mathrm{CoTPyP} / \mathrm{Au}_{13}$ geometric configuration, where the color scale indicates the electron transfer induced by $\mathrm{O}_{2}$ adsorption. The electron transfer to Co is 0.20 $\mathrm{e}^{-}$

of $0.1-0.2 \mathrm{eV}$ and large $\mathrm{Co}-\mathrm{O}$ distances with the $\mathrm{O}-\mathrm{O}$ bond lying fat, parallel to the macrocycle. Instead, the presence of Au stabilizes the system (Fig. 4), where the distance between the $\mathrm{Co}$ and the closest oxygen atom is $2.2 \AA$, the $\mathrm{O}-\mathrm{O}$ bond length increases from $1.23 \AA$ (in the gas phase) to $1.27 \AA$, and the $\mathrm{Co}-\mathrm{O}-\mathrm{O}$ angle is $118^{\circ}$ (Fig. 4a). We have also tested the sensitivity of this result on the choice of the DFT $+\mathrm{U}$ method: a simple PBE calculation gives an $\mathrm{O}_{2}$ adsorption energy of $0.71 \mathrm{eV}$, showing that this is a robust result. The presence of the ligand induces small changes in the CoTPyP geometry, with a small rotation of the pyridyl groups and a decrease of the Co-Au distance from 2.63 to $2.56 \AA$. The Co is lifted with respect to the macrocycle average plane by less than $0.1 \AA$. The electron charge transfer, projected on the single atoms, associated with the bonding of the adduct is shown in Fig. 4 by means of a color map. It is found that $\mathrm{O}_{2}$ adsorption causes an electron density depletion from the $\mathrm{Au}_{13}$ cluster $\left(-0.30 \mathrm{e}^{-}\right)$and from the pyrrolic $\mathrm{N}$ atoms $\left(-0.17 \mathrm{e}^{-}\right)$, corresponding to an electron density increase in the Co atom $\left(+0.20 \mathrm{e}^{-}\right)$and to $\mathrm{e}^{-}$transfer into the $\mathrm{O}_{2}$ molecule. The strong experimental indication of a drop of the nonresonant SFG amplitude upon $\mathrm{O}_{2}$ adsorption interestingly couples with the calculated charge depletion from the Au cluster to the oxygen adduct through the porphyrin plane. Indeed, the proximal $\mathrm{O}$ gains $0.09 \mathrm{e}^{-}$, while the distal oxygen atom gains $0.13 \mathrm{e}^{-}$. In this way the $\mathrm{O}_{2}$ ligand displays a dipole of $0.11 \mathrm{D}$ with a significant projection along the normal to the porphyrin plane, thus fulfilling the selection rules in order for the $\nu(\mathrm{O}-\mathrm{O})$ vibrational resonance to be SFG active. To make a comparison, the dipole of heteronuclear carbon monoxide, which notoriously gives a strong SFG signal [21, 22], is of the same order of magnitude. In Fig. 3a we report the corresponding calculated DOS projected on the two oxygen atoms ( $2 p$ levels) and on the Co $3 d \mathrm{z}^{2}, \mathrm{zx}$, and zy orbitals. There is evidence of a $\operatorname{Co}\left(3 d_{z} 2\right)-\mathrm{O}(2 p)$ overlap yielding covalent bonding at -7.9 and $-1.0 \mathrm{eV}$ with a strong contribution associated with the charge transfer from $\mathrm{Au}$ through the macrocycle plane (trans-effect) and the formation of a shared $\mathrm{Au}-\mathrm{Co}-\mathrm{O}_{2}$ peak in the DOS at $-7.9 \mathrm{eV}$ from the Fermi level (Fig. 5a). By comparing geometric and electronic structures, we understand that the $\mathrm{Co}-\mathrm{O}_{2}$ bond consists of a dative $\sigma(\mathrm{Co}-\mathrm{O})$ interaction between vacant $3 d_{\mathrm{z}} 2$ and $\pi^{*}\left(\mathrm{O}_{2}\right)$ and a weak $\pi$ interaction between $3 d_{\mathrm{yz}}$ and $\pi_{\perp}{ }^{*},{ }^{2}$ as already known in the literature for similar cobalt and iron tetrapyrroles $[6,58,70-72]$. The main fingerprint of this chemical bond is given by the adsorption geometry of $\mathrm{O}_{2}$, which forms a Co-O-O angle of $118^{\circ}$. This is a typical configuration in many oxygen-bonding biological tetrapyrrolebased proteins and enzymes, where the $\mathrm{M}-\mathrm{O}-\mathrm{O}$ angle varies in the $110-160^{\circ}$ range [6]. This bonding angle originates from the geometry of the $\pi^{*}\left(\mathrm{O}_{2}\right)$, which, in order to vertically align with the Co $3 d_{z} 2$, requires a tilting of the dioxygen molecule as it can be observed in Fig. 5b, where the isodensity surface of the state corresponding to the peak in the DOS at $0.4 \mathrm{eV}$ below the Fermi level is plotted. Interestingly, it can be noticed that also one of the $\mathrm{N}$ atoms of the macrocycle participates in the oxygen bonding (see Fig. 5c). We also find that the total $\mathrm{O}_{2}$ magnetization is lowered upon adsorption by $15 \%$ with respect to the gas-phase value $(\mathrm{S}=1)$. This is a key point in the activation process of $\mathrm{O}_{2}$. The oxygen molecule ground state is indeed a triplet, poorly reactive configuration with other small, common molecules that are in a singlet state: the most stable $\mathrm{O}_{2}$ configuration is $0.93 \mathrm{eV}$ lower in energy with respect to the singlet state with paired spin [73]. In order to promote the molecule's activation for further reaction, biomimetic catalysts need to

\footnotetext{
$\pi^{*}$ has the lobes-plane perpendicular to the molecular plane.
} 


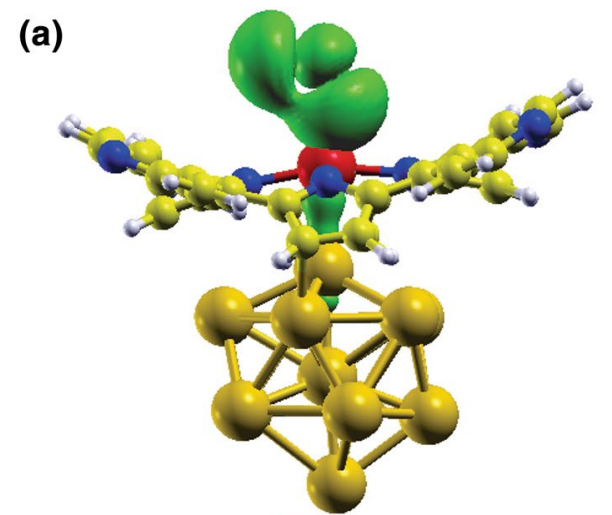

(b)

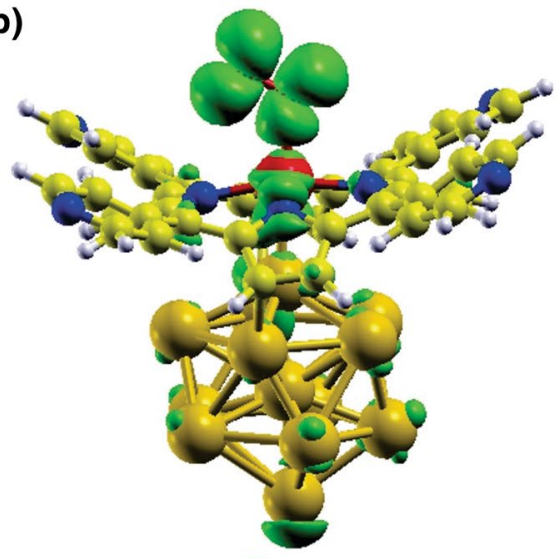

(c)

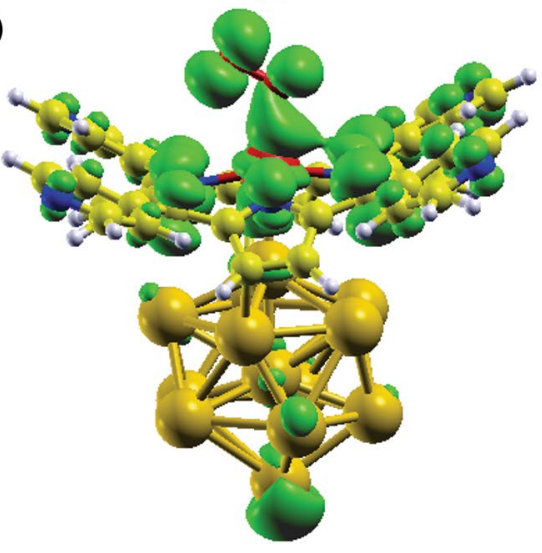

Fig. 5 Isodensity surfaces (green) of the electronic states of the $\mathrm{O}_{2}$-CoTPyP/Au $/ 3$ system corresponding to the peaks in the DOS indicated in Fig. 3 at 7.9 (a), 1.0 (b), and $0.4 \mathrm{eV}$ (c) below the Fermi level and obtained from the ab initio DFT simulations; the plots put in evidence the trans-effect and the origin of the $\mathrm{Co}-\mathrm{O}-\mathrm{O}$ bonding angle, respectively; color legend: yellow $(\mathrm{C})$, white $(\mathrm{H})$, blue $(\mathrm{N})$, gold $(\mathrm{Au})$, red $(\mathrm{Co})$

provide electron-donor sites where the molecule can bind [8] electron donation can take place, and reactive superoxo $\left(\mathrm{O}_{2}{ }^{-}\right)$or peroxo $\left(\mathrm{O}_{2}{ }^{2-}\right)$ species are formed to undergo the $2 \mathrm{e}^{-}$or the $4 \mathrm{e}^{-}$reduction processes, depending on the system $[74,75]$. Spin inversion is necessary for $\mathrm{O}_{2}$ activation since, even if ORR processes can be largely exothermic, spin-forbidden transitions contribute with high activation barriers.
Most transition metals offer unpaired electrons, both in the ground state or in almost degenerate excited states, allowing reaction with triplet $\mathrm{O}_{2}$, and spin-orbit coupled configurations, thus providing a quantum mechanical pathway to promote spin inversion [73], possibly exploiting di-radical intermediates for reaction [6].

\section{Conclusions}

By means of a combined experimental and computational approach, we have therefore shown that the biomimetic CoTPyP MOF grown on the Au(111) surface well-reproduces the main features of $\mathrm{O}_{2}$ bonding and activation typical of the biochemical environment. By means of a surface transeffect, the Co and macrocycle electronic configurations are tuned in order to provide the charge transfer that is required for the weakening of the $\mathrm{O}-\mathrm{O}$ bond and the initial modification of its spin configuration. The spectroscopic information is obtained in situ at close-to-ambient conditions thanks to the non-linear IR-Vis SFG approach, thus opening the way to a deeper comprehension of the initial ORR steps at synthetic 2D biomimetic systems.

Acknowledgments Open access funding provided by Università degli Studi di Trieste within the CRUI-CARE Agreement. F.A., M.S, and E.V. acknowledge financial support from Italian MIUR through project PRIN 2017KFY7XF.

Open Access This article is licensed under a Creative Commons Attribution 4.0 International License, which permits use, sharing, adaptation, distribution and reproduction in any medium or format, as long as you give appropriate credit to the original author(s) and the source, provide a link to the Creative Commons licence, and indicate if changes were made. The images or other third party material in this article are included in the article's Creative Commons licence, unless indicated otherwise in a credit line to the material. If material is not included in the article's Creative Commons licence and your intended use is not permitted by statutory regulation or exceeds the permitted use, you will need to obtain permission directly from the copyright holder. To view a copy of this licence, visit http://creativecommons.org/licenses/by/4.0/.

\section{References}

1. Yang XF, Wang A, Qiao B, Li J, Liu J, Zhang T (2013) Singleatom catalysts: a new frontier in heterogeneous catalysis. Acc Chem Res 46(8):1740-1748

2. Vesselli E (2020) Tetrapyrroles at near-ambient pressure: porphyrins and phthalocyanines beyond the pressure gap. J Phys Mater 3:022002. https://doi.org/10.1088/2515-7639/ab7ab2

3. Kadish KM, Smith KM, Guilard R (2003) The porphyrin handbook. Elsevier, Amsterdam

4. Meunier B (1992) Metalloporphyrins as versatile catalysts for oxidation reactions and oxidative DNA cleavage. Chem Rev 92:1411-1456. https://doi.org/10.1021/cr00014a008 
5. Zhang W, Lai W, Cao R (2017) Energy-related small molecule activation reactions: oxygen reduction and hydrogen and oxygen evolution reactions catalyzed by porphyrin- and corrole-based systems. Chem Rev 117:3717-3797. https://doi.org/10.1021/acs. chemrev.6b00299

6. Huang X, Groves JT (2018) Oxygen activation and radical transformations in Heme proteins and metalloporphyrins. Chem Rev 118:2491-2553. https://doi.org/10.1021/acs.chemrev.7b00373

7. Gutzler R, Stepanow S, Grumelli D et al (2015) Mimicking Enzymatic Active Sites on Surfaces for Energy Conversion Chemistry. Acc Chem Res 48:2132-2139. https://doi.org/10.1021/acs.accou nts.5b00172

8. Pereira MM, Dias LD, Calvete MJF (2018) Metalloporphyrins: bioinspired oxidation catalysts. ACS Catal. https://doi. org/10.1021/acscatal.8b01871

9. Costas M, Mehn MP, Jensen MP, Que L (2004) Dioxygen activation at mononuclear nonheme iron active sites: enzymes, models, and intermediates. Chem Rev 104:939-986. https://doi. org/10.1021/cr020628n

10. Lee J, Farha OK, Roberts J et al (2009) Metal-organic framework materials as catalysts. Chem Soc Rev 38:1450-1459. https://doi. org $/ 10.1039 / \mathrm{b} 807080 \mathrm{f}$

11. Shao M, Chang Q, Dodelet J-P, Chenitz R (2016) Recent advances in electrocatalysts for oxygen reduction reaction. Chem Rev 116:3594-3657. https://doi.org/10.1021/acs.chemrev.5b00462

12. She ZW, Kibsgaard J, Dickens CF et al (2017) Combining theory and experiment in electrocatalysis: insights into materials design. Science. https://doi.org/10.1126/science.aad4998

13. Nie Y, Li L, Wei Z (2015) Recent advancements in Pt and Pt-free catalysts for oxygen reduction reaction. Chem Soc Rev 44:21682201. https://doi.org/10.1039/c4cs00484a

14. Cao R, Lai W, Du P (2012) Catalytic water oxidation at single metal sites. Energy Environ Sci 5:8134. https://doi.org/10.1039/ c2ee21494f

15. Koper MTM (2011) Thermodynamic theory of multi-electron transfer reactions: Implications for electrocatalysis. J Electroanal Chem 660:254-260. https://doi.org/10.1016/j.jelec hem.2010.10.004

16. Janet JP, Zhao Q, Ioannidis EI, Kulik HJ (2017) Density functional theory for modelling large molecular adsorbate-surface interactions: a mini-review and worked example. Mol Simul 43:327-345. https://doi.org/10.1080/08927022.2016.1258465

17. Wurster B, Grumelli D, Hötger D et al (2016) Driving the Oxygen Evolution Reaction by Nonlinear Cooperativity in Bimetallic Coordination Catalysts. J Am Chem Soc 138:3623-3626. https:// doi.org/10.1021/jacs.5b10484

18. Yoshimoto S, Inukai J, Tada A et al (2004) Adlayer structure of and electrochemical $\mathrm{O}_{2}$ reduction on cobalt porphine-modified and cobalt octaethylporphyrin-modified $\mathrm{Au}(111)$ in $\mathrm{HClO}_{4}$. J Phys Chem B 108:1948-1954. https://doi.org/10.1021/jp0366421

19. Xiang S, He Y, Zhang Z et al (2012) Microporous metal-organic framework with potential for carbon dioxide capture at ambient conditions. Nat Commun. https://doi.org/10.1038/ncomms 1956

20. Yazaydin AO, Snurr RQ, Park T-H et al (2009) Screening of metal-organic frameworks for carbon dioxide capture from flue gas using a combined experimental and modeling approach. J Am Chem Soc 131:18198-18199. https://doi.org/10.1021/ja9057234

21. Corva M, Ferrari A, Rinaldi M et al (2018) Vibrational fingerprint of localized excitons in a two-dimensional metal-organic crystal. Nat Commun 9:4703. https://doi.org/10.1038/s41467-018-07190 $-1$

22. Corva M, Mohamed F, Tomsic E et al (2019) Learning from nature: charge transfer and carbon dioxide activation at single, biomimetic Fe sites in tetrapyrroles on graphene. J Phys Chem C 123:3916-3922. https://doi.org/10.1021/acs.jpcc.8b11871
23. Hieringer W, Flechtner K, Kretschmann A et al (2011) The surface trans effect: influence of axial ligands on the surface chemical bonds of adsorbed metalloporphyrins. J Am Chem Soc 133:62066222. https://doi.org/10.1021/ja1093502

24. Rupprechter G, Weilach C (2007) Mind the gap! Spectroscopy of catalytically active phases. Nano Today 2:20-29. https://doi. org/10.1016/S1748-0132(07)70114-7

25. Salmeron M, Schlögl R (2008) Ambient pressure photoelectron spectroscopy: a new tool for surface science and nanotechnology. Surf Sci Rep 63:169-199. https://doi.org/10.1016/j.surfr ep.2008.01.001

26. Tian CS, Shen YR (2014) Recent progress on sum-frequency spectroscopy. Surf Sci Rep 69:105-131. https://doi.org/10.1016/j. surfrep.2014.05.001

27. Hötger D, Etzkorn M, Morchutt C et al (2019) Stability of metalloporphyrin networks under oxygen reduction and evolution conditions in alkaline media. Phys Chem Chem Phys 21:2587-2594. https://doi.org/10.1039/c8cp07463a

28. Yoshimoto S, Tada A, Suto K et al (2003) Adlayer structure and electrochemical reduction of $\mathrm{O}_{2}$ on self-organized arrays of cobalt and copper tetraphenyl porphines on a $\mathrm{Au}(111)$ surface. Langmuir 19:672-677. https://doi.org/10.1021/la026449i

29. Zheng S, Jiujun Z (2007) Density functional theory study of transitional metal macrocyclic complexes' dioxygen-binding abilities and their catalytic activities toward oxygen reduction reaction. J Phys Chem C 111:7084-7090. https://doi. org/10.1021/jp0671749

30. Mandal B, Chung JS, Kang SG (2018) Theoretical insight into M1TPyP-M2 (M1, M2 = Fe, Co) MOFs: correlation between electronic structure and catalytic activity extending to potentiality in capturing flue gases. J Phys Chem C 122:9899-9908. https ://doi.org/10.1021/acs.jpcc.8b00080

31. Balents L (2010) Spin liquids in frustrated magnets. Nature 464:199-208. https://doi.org/10.1038/nature08917

32. Son Y-W, Cohen ML, Louie SG (2006) Half-metallic graphene nanoribbons. Nature 444:347-349. https://doi.org/10.1038/natur e05180

33. Lee PA, Nagaosa N, Wen X-G (2006) Doping a Mott insulator: physics of high-temperature superconductivity. Rev Mod Phys 78:17-85. https://doi.org/10.1103/RevModPhys.78.17

34. Anderson PW (1987) The resonating valence bond state in $\mathrm{La}_{2} \mathrm{CuO}_{4}$ and superconductivity. Science 235:1196-1198. https ://doi.org/10.1126/science.235.4793.1196

35. Murphy BE, Krasnikov SA, Sergeeva NN et al (2014) Homolytic cleavage of molecular oxygen by manganese porphyrins supported on ag(111). ACS Nano 8:5190-5198. https://doi.org/10.1021/ $\mathrm{nn} 501240 \mathrm{j}$

36. Shen YR (1989) Surface properties probed by second-harmonic and sum-frequency generation. Nature 337:519-525. https://doi. org/10.1038/337519a0

37. Corva M, Vesselli E (2016) Room temperature carbonylation of iron-phthalocyanines adsorbed on a single crystal metal surface: an in situ SFG investigation at near-ambient pressure. J Phys Chem C 120:22298-22303. https://doi.org/10.1021/acs. jpcc.6b05356

38. Dalstein L, Revel A, Humbert C, Busson B (2018) Nonlinear optical response of a gold surface in the visible range: a study by two-color sum-frequency generation spectroscopy. I. Experimental determination. J Chem Phys 148:134701. https://doi. org/10.1063/1.5021553

39. Busson B, Dalstein L (2018) Nonlinear optical response of a gold surface in the visible range: a study by two-color sum-frequency generation spectroscopy. III. Simulations of the experimental SFG intensities. J Chem Phys 149:154701. https://doi. org/10.1063/1.5047098 
40. Busson B, Dalstein L (2018) Nonlinear optical response of a gold surface in the visible range: a study by two-color sum-frequency generation spectroscopy. II.Model for metal nonlinear susceptibility. J Chem Phys 149:034701. https://doi.org/10.1063/1.5027154

41. Corva M, Feng Z, Dri C et al (2016) Carbon dioxide reduction on $\operatorname{Ir}(111)$ : stable hydrocarbon surface species at near-ambient pressure. Phys Chem Chem Phys 18:6763-6772. https://doi. org/10.1039/C5CP07906C

42. Hayashi M, Lin SH, Raschke MB, Shen YR (2002) A molecular theory for doubly resonant IR-UV-vis sum-frequency generation. J Phys Chem A 106:2271-2282. https://doi.org/10.1021/jp012 6331

43. Bonn M, Hess C, Roeterdink WG et al (2004) Dephasing of vibrationally excited molecules at surfaces: $\mathrm{CO} / \mathrm{Ru}(001)$. Chem Phys Lett 388:269-273. https://doi.org/10.1016/j.cplett.2004.03.022

44. Giannozzi P, Baroni S, Bonini N et al (2009) QUANTUM ESPRESSO: a modular and open-source software project for quantum simulations of materials. J Phys Condens Matter 21:395502. https://doi.org/10.1088/0953-8984/21/39/395502

45. Giannozzi P, Andreussi O, Brumme T et al (2017) Advanced capabilities for materials modelling with Quantum ESPRESSO. J Phys Condens Matter 29:465901. https://doi.org/10.1088/1361-648X/ aa8f79

46. Vanderbilt D (1990) Soft self-consistent pseudopotentials in a generalized eigenvalue formalism. Phys Rev B 41:7892-7895. https:// doi.org/10.1103/PhysRevB.41.7892

47. Perdew JP, Burke K, Ernzerhof M (1996) Generalized gradient approximation made simple. Phys Rev Lett 77:3865-3868. https:// doi.org/10.1103/PhysRevLett.77.3865

48. Anisimov VI, Zaanen J, Andersen OK (1991) Band theory and Mott insulators: Hubbard U instead of Stoner I. Phys Rev B 44:943-954. https://doi.org/10.1103/PhysRevB.44.943

49. Chen J, Wu X, Selloni A (2011) Electronic structure and bonding properties of cobalt oxide in the spinel structure. Phys Rev B 83:245204. https://doi.org/10.1103/PhysRevB.83.245204

50. Grimme S, Antony J, Ehrlich S, Krieg H (2010) A consistent and accurate ab initio parametrization of density functional dispersion correction (DFT-D) for the 94 elements H-Pu. J Chem Phys 132:154104. https://doi.org/10.1063/1.3382344

51. Löwdin P-O (1955) Quantum theory of many-particle systems. I. Physical interpretations by means of density matrices, natural spinorbitals, and convergence problems in the method of configurational interaction. Phys Rev 97:1474-1489. https://doi.org/10.1103/PhysR ev.97.1474

52. Araki K, Santos PS, de Oliveira LFCC, Toma HE (1995) Resonance Raman spectra of a supramolecular species containing four ruthenium(II)-bipyridine complexes attached to zinctetrapyridyl porphynate. Spectrosc Lett 28:119-126. https://doi. org/10.1080/00387019508011660

53. Blom N, Odo J, Nakamoto K, Strommen DP (1986) Resonance Raman studies of metal tetrakis(4-N-methylpyridyl)porphine: band assignments, structure-sensitive bands, and species equilibria. J Phys Chem 90:2847-2852. https://doi.org/10.1021/j100404a015

54. Popovici S, Leyffer W, Holze R (1998) The mechanism of dioxygen reduction at iron meso-tetrakis (pyridyl) porphyrin: a spectroelectrochemical study. J Porphyr Phthalocyanines 2:249-260. https:// doi.org/10.1002/(SICI)1099-1409(199805/06)2:3<249:AID-JPP77 $>3.3 . \mathrm{CO} ; 2-\mathrm{V}$

55. Schmidt J, Stuhlmann C, Ibach H (1993) Oxygen adsorption on the $\operatorname{Pt}(110)(1 \times 2)$ surface studied with EELS. Surf Sci 284:121-128. https://doi.org/10.1016/0039-6028(93)90530-W

56. Vattuone L, Gambardella P, Valbusa U, Rocca M (1997) HREELS study of $\mathrm{O}_{2}$ molecular chemisorption on $\mathrm{Ag}(001)$. Surf Sci 377379:671-675. https://doi.org/10.1016/S0039-6028(96)01471-9
57. Wong SF, Boness MJW, Schulz GJ (1973) Vibrational excitation of $\mathrm{O}_{2}$ by electron impact above $4 \mathrm{eV}$. Phys Rev Lett 31:969-972. https ://doi.org/10.1103/PhysRevLett.31.969

58. Kozuka M, Nakamoto K (1981) Vibrational studies of (tetraphenylporphyrinato)cobalt(II) and its adducts with $\mathrm{CO}, \mathrm{NO}$, and $\mathrm{O}_{2}$ in gas matrices. J Am Chem Soc 103:2162-2168. https://doi.org/10.1021/ ja00399a004

59. Backx C, De Groot CPM, Biloen P (1981) Adsorption of oxygen on $\mathrm{Ag}(110)$ studied by high resolution ELS and TPD. Surf Sci 104:300-317. https://doi.org/10.1016/0039-6028(81)90137-0

60. Vattuone L, Valbusa U, Rocca M (1994) Coverage dependence of the O-Ag (110) vibration. Surf Sci 317:L1120-L1123. https://doi. org/10.1016/0039-6028(94)90246-1

61. Nguyen D, Kang G, Chiang N et al (2018) Probing molecularscale catalytic interactions between oxygen and cobalt phthalocyanine using tip-enhanced Raman spectroscopy. J Am Chem Soc 140:5948-5954. https://doi.org/10.1021/jacs.8b01154

62. Woodruff P (2016) Modern techniques of surface science. Cambridge University Press, Cambridge

63. Baletto F, Ferrando R (2005) Structural properties of nanoclusters: Energetic, thermodynamic, and kinetic effects. Rev Mod Phys 77:371-423. https://doi.org/10.1103/RevModPhys.77.371

64. Auwärter W, Seufert K, Klappenberger F et al (2010) Site-specific electronic and geometric interface structure of Co-tetraphenylporphyrin layers on $\operatorname{Ag}(111)$. Phys Rev B 81:245403. https://doi. org/10.1103/PhysRevB.81.245403

65. Müllegger S, Schöfberger W, Rashidi M et al (2009) Spectroscopic STM studies of single gold(III) porphyrin molecules. J Am Chem Soc 131:17740-17741. https://doi.org/10.1021/ja908157j

66. Müllegger S, Rashidi M, Schöfberger W, Koch R (2018) Singlemolecule chemical reduction induced by low-temperature scanning tunneling microscopy: a case study of gold-porphyrin on $\mathrm{Au}(111)$. Surf Sci 678:157-162. https://doi.org/10.1016/j.susc.2018.04.018

67. Senge M, Ryan A, Letchford K et al (2014) Chlorophylls, symmetry, chirality, and photosynthesis. Symmetry (Basel) 6:781-843. https:// doi.org/10.3390/sym6030781

68. Zhang Q, Kuang G, Pang R et al (2015) Switching molecular Kondo effect via supramolecular interaction. ACS Nano 9:12521-12528. https://doi.org/10.1021/acsnano.5b06120

69. Barlow DE, Scudiero L, Hipps KW (2004) Scanning tunneling microscopy study of the structure and orbital-mediated tunneling spectra of cobalt(II) phthalocyanine and cobalt(II) tetraphenylporphyrin on $\mathrm{Au}(111)$ : Mixed composition films. Langmuir 20:44134421. https://doi.org/10.1021/la0358791

70. Chen $\mathrm{H}$, Ikeda-Saito $\mathrm{M}$, Shaik $\mathrm{S}$ (2008) Nature of the $\mathrm{Fe}-\mathrm{O}_{2}$ bonding in oxy-myoglobin: effect of the protein. J Am Chem Soc 130:1477814790. https://doi.org/10.1021/ja805434m

71. Ponce I, Silva JF, Oñate R et al (2012) Enhancement of the catalytic activity of fe phthalocyanine for the reduction of $\mathrm{O}_{2}$ anchored to $\mathrm{Au}(111)$ via conjugated self-assembled monolayers of aromatic thiols as compared to Cu phthalocyanine. J Phys Chem C 116:15329_ 15341. https://doi.org/10.1021/jp301093q

72. Wayland BB, Minkiewicz JV, Abd-Elmageed ME (1974) Spectroscopic studies for tetraphenylporphyrincobalt(II) complexes of $\mathrm{CO}$, $\mathrm{NO}, \mathrm{O}_{2}, \mathrm{RNC}$, and (RO)3P, and a bonding model for complexes of $\mathrm{CO}, \mathrm{NO}$, and $\mathrm{O}_{2}$ with cobalt(II) and iron(II) porphyrins. J Am Chem Soc 96:2795-2801. https://doi.org/10.1021/ja00816a022

73. Jensen KP, Ryde U (2004) How $\mathrm{O}_{2}$ binds to heme. reasons for rapid binding and spin inversion. J Biol Chem 279:14561-14569. https:// doi.org/10.1074/jbc.M314007200

74. Mingos DMP (1971) Geometries of Dioxygen (O2), Superoxo (O2-) and Peroxo (O22-) Complexes. Nat Phys Sci 230:154-156. https://doi.org/10.1038/physci230154a0

75. Kim E, Helton ME, Wasser IM et al (2003) Superoxo, -peroxo, and -oxo complexes from heme $/ \mathrm{O}_{2}$ and heme- $\mathrm{Cu} / \mathrm{O}_{2}$ reactivity: copper 
ligand influences in cytochrome c oxidase models. Proc Natl Acad Sci USA 100:3623-3628. https://doi.org/10.1073/pnas.0737180100

76. Burke JM, Kincaid JR, Spiro TG (1978) Resonance Raman spectra and vibrational modes of iron(III) tetraphenylporphine $\mu$-Oxo dimer. Evidence for phenyl interaction and lack of dimer splitting. J Am Chem Soc 100:6077-6083. https://doi.org/10.1021/ja00487a017

77. Burke JM, Kincaid JR, Spiro TG et al (1978) Structure-Sensitive Resonance Raman Bands of Tetraphenyl and "Picket Fence" Porphyrin-Iron Complexes, Including an Oxyhemoglobin Analogue. J Am Chem Soc 100:6083-6088. https://doi.org/10.1021/ja00487a01 8
78. Simic-Glavaski B, Zecevic S, Yeager EB (1983) Spectroscopic and electrochemical studies of transition metal tetrasulfonated phthalocyanines: 2-resonant raman spectra of aqueous solutions of cobalt and iron tetrasulfonated phthalocyanines. J Raman Spectrosc 14:338-341. https://doi.org/10.1002/jrs.1250140509

Publisher's Note Springer Nature remains neutral with regard to jurisdictional claims in published maps and institutional affiliations. 